\title{
Book review: Mensch - Umwelt - Klimawandel. Globale Herausforderungen und lokale Resillienz im Schweizer Hochgebirge
}

\author{
Dominik Siegrist \\ Institut für Landschaft und Freiraum, OST Ostschweizer Fachhochschule, Rapperswil, Schweiz \\ Correspondence: Dominik Siegrist (dominik.siegrist@ost.ch)
}

Received: 15 January 2021 - Published: 1 February 2021

Reichel, C.: Mensch-Umwelt-Klimawandel. Globale Herausforderungen und lokale Resilienz im Schweizer Hochgebirge, transcript, Bielefeld, 298 S., ISBN: 978-3-8376-46962, EUR 40,00, 2020.

Mit seinem Buch über Mensch, Umwelt und Klimawandel legt der Berliner Geograf Christian Reichel einen aktuellen Beitrag zur Alpenforschung vor. Auf den ersten Blick erscheint die Beschäftigung mit der Thematik Umwelt und Klima nicht neu, wurde diese doch bereits in den letzten Jahrzehnten in vielen Forschungen immer wieder verfolgt. Zum Beispiel bereits im MAB-Programm der UNESCO in den 1980er-Jahren oder im Rahmen des schweizerischen Nationalen Forschungsprogramms NFP 48 ,Landschaften und Lebensräume der Alpen“ (2002-2007). Bereits in diesen Forschungen erhielten interdisziplinäre Ansätze einen hohen Stellenwert und Umwelt-, Sozial- und Wirtschaftswissenschaftler spannten zusammen. Wer sich die Mühe nimmt, Reichels Buch zu lesen, wird darin jedoch Erkenntnisse und Problemlösungsansätze finden, die in dieser Form durchaus als neu und innovativ gelten können. Der Autor stellt nämlich die Folgen des sich derzeit massiv verstärkenden Klimawandels auf die Berggebiete und weitere gravierende Umweltveränderungen ins Zentrum seiner Forschungen. Und schafft dabei Bezüge zur aktuellen Resilienzforschung, zu modernen digitalen Techniken und zur Partizipation der Bevölkerung im Berggebiet.

In seiner Publikation verfolgt Christian Reichel die Absicht aufzuzeigen, dass sich die Auswirkungen destruktiver Mensch-Umwelt-Beziehungen in den Alpen nur hinreichend verstehen lassen, wenn man auch deren lokale Einbettung berücksichtigt. Im Fokus stehen damit die engen Bezüge zwischen dem alltäglichen Wirtschaften der Bevölkerung, insbesondere in der Berglandwirtschaft, und deren teils über lange
Zeit überliefertem lokalen Handlungs-, Erfahrungs- und Umweltwissen. Untersucht wird die umfassende Forschungsfrage, über welches lokale Umweltwissen die Bergbäuerinnen und Bergbauern zur Stärkung der Resilienz gegenüber potenziellen Naturgefahren und bezüglich einer nachhaltigen Ressourcennutzung verfügen. Dabei interessiert den Autoren, welche Faktoren das lokale Umweltwissen prägen beziehungsweise wie es sich durch exogene und endogene Einflüsse auf das Tal mit der Zeit verändert.

Auf der theoretischen Ebene bezieht sich Reichel auf einen sozial-ökologischen Systemansatz, wobei er sich zwangsläufig im Spannungsfeld zwischen Interdisziplinarität in ihrer ganzen Komplexität und der Gefahr einer durch Nähe zur Praxis geforderten Reduktion bewegt. Dass sein empirisches Forschungsobjekt eine Region in den Alpen darstellt, ist einerseits dem persönlichen Bezug des Autors zu dieser Grossregion geschuldet, andererseits seinem Interesse an der aktuellen Debatte über die Zukunft der Berggebiete angesichts der Bedrohung durch den gegenwärtigen verstärkten ökologischen, sozialen und wirtschaftlichen Wandel. Dabei stellt der Autor nicht in Abrede, dass seine vertiefte Beschäftigung mit Fragen der Naturwahrnehmung und Naturrezeption der Alpen durchaus auch einen Bezug zur Projektionsfläche Alpen aufweist, der auch er sich nicht vollständig entziehen kann.

Die empirischen Untersuchungen von Reichel beziehen sich schwerpunktmässig auf das Safiental im Schweizer Kanton Graubünden, ein abgelegenes alpines Gebiet mit geringer Bevölkerungsdichte, geprägt durch Bergland- und Forstwirtschaft und durch extensiven Tourismus. Vergleichend dazu wird eine Untersuchung des Bergvolks der Toraja Masupu in Süd-Sulawesi (Indonesien) beigezogen, wobei eine Reihe von Parallelen, aber auch viele Unterschiede festge- 
stellt werden (z.B. im Zusammenhang mit der unterschiedlichen Bedeutung der Religion). Im Rahmen der Fallstudien kommt ein innovatives Set von qualitativen Forschungsmethoden zur Anwendung, angefangen bei der teilnehmenden Beobachtung über halbstrukturierte Leitfadeninterviews und Gruppeninterviews, strukturierte Begehungen, partizipative Kartierungen bis hin zu multimedialen Kartierungen in Kombination mit Film und Fotografie.

So vielfältig die angewendeten Methoden, so reichhaltig sind die Ergebnisse von Reichels Erhebungen. Davon ausgehend konstatiert er für das Safiental zwei hauptsächliche Triebkräfte des sozial-ökologischen Wandels: erstens den umfassenden Strukturwandel in der Landwirtschaft und zweitens die verschiedenen Umweltveränderungen. So müssen immer weniger Landwirte - angetrieben durch die Subventionspolitik des Bundes - immer grössere Flächen bewirtschaften, was eine starke Mechanisierung mit deutlich negativen Folgen für den Umgang mit der Umwelt zur Folge hat. Insbesondere lokales Erfahrungs- und Umweltwissen sei dadurch bei der jüngeren Generation weitgehend verloren gegangen, bemerkt der Autor. Dabei führten die Folgen des Klimawandels zu einer Zunahme der Naturgefahren, die immer schwieriger zu kontrollieren sei. Das Zusammentreffen dieser beiden Phänomene, der Zunahme der neuen, oft unberechenbaren Umweltveränderungen und des Verlusts an lokalem Umwelt- und Erfahrungswissen, münde in einem ,doppelten Risiko“. Reichel warnt deshalb davor, dass sich für das untersuchte Gebiet multiple Risiken aus der komplexen Verbindung von klimabedingten Risiken und den langfristigen sozial-ökologischen und sozioökonomischen Auswirkungen eines umfassenden Strukturwandels in der Landwirtschaft ergeben.

Mit Blick auf nachhaltige Klimaanpassungsstrategien verneint der Autor zunächst ein unausweichliches Dilemma destruktiver Ressourcennutzung und Sozialbrache. Er sieht durchaus Möglichkeiten einer nachhaltigen Wirtschaftsweise, in deren Rahmen die Bevölkerung mit dem Wandel und den Gefahren proaktiv umgeht und lokales Umwelt- und Erfahrungswissen für die Stärkung der eigenen Resilienz miteinbezieht. Im Rahmen eines ,integrierten Risikoansatzes“ bedürfe es allerdings einer Prioritätensetzung, welche Risiken akzeptiert werden sollen und welche nicht. Solche durchaus sensiblen Fragen könnten in einer Kombination aus TopDown- und Bottom-Up-Ansätzen unter Einbezug der Bevölkerung und von Fachleuten geklärt werden.

Eine von Reichel exemplarisch für das Naturgefahrenmanagement eingesetzte Praxismethode ist die Multimediakartierung. Am Beispiel des Safientals (mit dem thematischen Schwerpunkt des Strukturwandels in der Landwirtschaft) stellt er unterschiedliche qualitative Karten strukturiert dar bzw. führt diese zusammen. Dabei bezieht er auch Filme, Audioaufzeichnungen, Notizen und partizipative Karten mit ein. Als Vorteile dieser Vorgehensweise führt der Autor die Dokumentation lokalen Wissens, die Partizipation der lokalen Bevölkerung, die inter- und transdisziplinäre Zusammenar- beit sowie die leichtere Kommunikation zwischen Experten und Bevölkerung an.

Christian Reichel legt mit seinem Buch eine interessante Untersuchung über eine bezüglich der Zukunft der Berggebiete zentrale Fragestellung vor. Seine detaillierte qualitative Bestandesaufnahme am Beispiel eines peripheren Bergtals fördert zu Tage, dass Umwelt- und Klimaveränderungen in den Alpen nicht mehr nur ein Zukunftsthema sind, sondern dass sich daraus bereits heute massive Probleme für eine Talschaft wie das Safiental ergeben. Mit dem Ansatz einer partizipativ angelegten Multimediakartierung stellt er einen methodischen Ansatz zur Diskussion, der künftig bei der Bewältigung der sich weiter verschärfenden Probleme hilfreich sein dürfte. Vielleicht kann damit lokales Umwelt- und Erfahrungswissen tatsächlich für die Praxis künftiger Generationen gesichert werden.

Allerdings wird die Anwendung innovativer multimedialer Dokumentationsmethoden allein für die Bewältigung der in den Berggebieten anstehenden Probleme nicht ausreichen, wenn gleichzeitig nicht auch die (politische) Bereitschaft der Bevölkerung gefördert wird, die neuen Probleme mit neuen Ansätzen anzugehen. In vielen Talschaften der Alpen ist heute eher das Gegenteil zu beobachten, indem mit zunehmenden Problemen und Herausforderungen die Bereitschaft der Bevölkerung tendenziell sinkt, grundsätzlich neue Pfade einzuschlagen. 